\title{
Dual kidney transplantation offers a safe and effective way to use kidneys from deceased donors older than 70 years
}

\author{
Kyo Won Lee ${ }^{1}$, Jae Berm Park ${ }^{1 *}$ D, So Ra Cha², Seo Hee Lee ${ }^{1}$, Young Jae Chung ${ }^{1}$, Heejin Yoo ${ }^{3}$, Kyunga Kim ${ }^{3}$ and \\ Sung Joo Kim
}

\begin{abstract}
Purpose: Dual kidney transplantation (DKT) offers a way to extend the use of kidneys from expanded criteria donors (ECDs). Here, we compared the outcomes of DKT with those of single kidney transplantation from standard criteria donors (SCDs) and ECDs.

Methods: In 2014, we began performing DKT using both kidneys from deceased donors greater than 70 years of age with one of two risk factors: serum creatinine $(\mathrm{s} C \mathrm{r}$ ) level over $3.0 \mathrm{mg} / \mathrm{dl}$ or eGFR under $30 \mathrm{ml} / \mathrm{min}$. By 2017 , we had performed 15 DKTs. We compared the outcomes of the 15 DKT recipients with those of 124 patients who received a kidney from an SCD and 80 patients who received a kidney from an ECD.

Results: Compared with ECDs and SCDs, DKT donors were older, had a higher diabetes burden, and a higher sCr level $(p<0.01,<0.01$, and 0.03 , respectively). DKT recipients were also older and had a higher diabetes burden than recipients of kidneys from ECDs and SCDs ( $p<0.01$, both). DKT recipients had a lower nadir sCr and shorter duration to nadir sCr than single ECD KT recipients ( $p<0.01$ and 0.04 , respectively).

Conclusions: The survival rates of DKT grafts were compatible with those of single KT grafts. Therefore, DKT may be considered a suitable an option to expand the donor pool.
\end{abstract}

Keywords: Expanded criteria donor, Old age donor, Dual kidney transplantation

\section{Background}

The rapidly growing incidence of chronic kidney disease and limited supply of donor organs has led to an increase in the number of patients awaiting kidney transplantation (KT). To overcome this organ shortage, kidneys from expanded criteria donors (ECD) or suboptimal donors are now widely used for transplantation. Importantly, the reported outcomes for KTs from ECDs are not inferior to those for KTs from standard criteria donors (SCDs) [1-3]. However, there remains a huge gap between the supply and demand for donor kidneys. According to data from Korean Network for Organ Sharing (KONOS), 7.9\% of kidneys from potential donors were discarded between 2010 and 2018. Kidneys from donors older than 60 or 70

\footnotetext{
* Correspondence: jbparkmd@gmail.com

${ }^{1}$ Department of Surgery, Samsung Medical Center, Sungkyunkwan University School of Medicine, 81 Irwon-ro, Gangnam-gu, Seoul 06351, South Korea Full list of author information is available at the end of the article
}

are more likely to be discarded (12.0 and $18.1 \%$, respectively). These high discard rates among older donors in their 60's and 70's are notable, since the proportion of such donors is increasing, accounting for 24.5 and $6.7 \%$ of Korean donors, in 2018, respectively. Dual kidney transplantation (DKT) offers a way to address the shortage of donor kidneys by reducing the organ discard rate [4]. Indeed, many centers have recently started to perform DKTs using their own selection criteria [5-10]. As of 2013, deceased donors in Korea could donate both kidneys to a single candidate when they were older than 70 and met at least one of the following criteria: 1) Estimated glomerular filtration rate (eGFR) calculated by MDRD equation less than $30 \mathrm{~mL} / \mathrm{min}$ without improvement, 2) Serum creatinine $(\mathrm{sCr})$ level higher than $3.0 \mathrm{mg} / \mathrm{dL}$ without improvement, or when a single kidney is refused by all other centers. 
Here, we describe a single center experience and outcomes of DKT using kidneys from extremely marginal donors with age over 70 and acute kidney injury.

\section{Methods}

\section{Study design}

This study was a retrospective single center historical cohort study and analyzed Samsung Medical Center electronic medical record and kidney transplantation database. We screened the records of 242 recipients who underwent deceased donor kidney transplantation (DDKT) between January 2014 and November 2017 in Samsung Medical Center. We excluded three pediatric recipients, four recipients who underwent en-bloc kidney transplantation, and 16 recipients who underwent multi-organ transplantation. The remaining 219 recipients included in the study were divided into three groups according to donor status. Group 1 ( $n=$ 124) consisted of patients who received a kidney from an SCD; Group $2(n=80)$ consisted of patients who received a kidney from an ECD; Group $3(n=15)$ consisted of patients who underwent DKT.

All data analyzed in the study were derived from our institution's electronic medical records and kidney transplantation database. The institutional review board of Samsung Medical Center approved this study protocol (SMC 2018-03-035) and waived the requirement for written informed consent.

\section{Donor selection criteria and definitions}

ECD was defined based on United Network for Organ Sharing (UNOS) criteria. ECDs consisted of deceased donors (DDs) older than 60 years and DDs 50 to 59 years of age who met two of the following criteria: (1) history of hypertension, (2) cerebrovascular accident as a cause of brain death, and (3) final pre-procurement $\mathrm{sCr}$ level > $1.5 \mathrm{mg} / \mathrm{dL}$ [11]. DKTs were attempted when the donor was older than 70 and met at least one of the following criteria: (1) an eGFR calculated by MDRD equation < 30 $\mathrm{mL} / \mathrm{min}$ without improvement or (2) an $\mathrm{sCr}$ level $>3.0$ $\mathrm{mg} / \mathrm{dL}$ without improvement or, regardless of donor age, when the single kidney was refused by all other centers. Kidney(s) were not accepted if they were grossly discolored or atrophied, or if the donor's $\mathrm{sCr}$ level had risen gradually for more than 5 days.

Urine leakage, ureteral stricture, post-operative bleeding, renal artery stenosis, and lymphocele requiring drainage were included as surgical complications. Graft failure was defined as the need for either permanent dialysis or re-transplantation. Delayed graft function (DGF) was defined as needing dialysis during the first week post-transplantation. eGFR values were calculated by the MDRD study equation.

\section{Surgical technique}

For DKT, we used a midline incision and implanted the graft kidneys in the intraperitoneal space separately on both sides. Anastomoses to the iliac vessels were performed separately on each side. Ureteroneocystostomies were performed bilaterally after revascularization of both kidneys using the Lich-Gregoir technique, with a double J stent for each ureter.

\section{Postoperative management}

We used rabbit anti-thymocyte globulin (rATG, $1.5 \mathrm{mg}$ / $\mathrm{kg}$, three doses on days $0,1,2$ ) as an induction immunosuppressive agent for recipients with donor-specific antiHLA antibodies (DSAs) or history of $\mathrm{KT}$, as well as in single ECD KT and DKT cases. Otherwise, we used an interleukin-2 receptor antagonist induction (Baxilixmab, $20 \mathrm{mg} / \mathrm{kg}$, two doses on days 0 and 4) as the induction immunosuppressive agent. A single dose of anti-CD20 monoclonal antibody (Rituximab, $375 \mathrm{mg} / \mathrm{m}^{2}$, single dose on day 0) was given to all recipients with DSAs. In all cases, the induction agent was ultimately decided according to the physician's preference.

Tacrolimus, mycophenolate, and steroids were used as maintenance immunosuppressive agents. Our detailed protocol for maintenance immunosuppressive agents, as well as infection prophylaxis and monitoring, has been previously described [12].

Protocol biopsies were performed 14 days and one year after KT. Biopsies were not performed for DKT recipients since the grafts were not fixed in the retroperitoneal space and the risk of post-biopsy bleeding was high. Biopsies were also performed in case of suspected acute rejection, such as a noted elevation in $\mathrm{sCr}$ level.

\section{Statistical analysis}

Continuous variables were analyzed with by the Kruskal Wallis test and post-hoc analysis was performed by the Wilcoxon rank sum test. Categorical variables were analyzed by Fisher's exact test. Graft and patient survival rates were obtained by Kaplan-Meier analysis and risk factor analysis was performed by Cox proportional-hazards regression model. Stepwise selection methods were applied to identify co-variables in the Cox proportional-hazards regression model (significance criteria 0.05 for entry and removal). The Generalized Estimating Equation (GEE) was applied to analyze repeated measurements for $\mathrm{sCr}$ and eGFR levels. Statistical analyses were performed using SAS version 9.4 (SAS Institute Inc., Cary, NC, USA).

\section{Results}

Donor and recipient characteristics

Donor characteristics are summarized in Table 1. The mean donor age, BMI, and pre-retrieval sCr level of the 
Table 1 Donor characteristics

\begin{tabular}{|c|c|c|c|c|c|c|}
\hline & $\operatorname{SCD}(n=124)$ & $\mathrm{ECD}(n=80)$ & DKT $(n=15)$ & $p$ value & SCD vs DKT & ECD vs DKT \\
\hline Age (year) & $42.6 \pm 12.0$ & $64.4 \pm 7.2$ & $74.5 \pm 5.1$ & $<0.01$ & $<0.01$ & $<0.01$ \\
\hline Sex (M:F) & $78: 46$ & $51: 29$ & $7: 8$ & 0.45 & & \\
\hline $\mathrm{BMI}\left(\mathrm{kg} / \mathrm{m}^{2}\right)$ & $23.5 \pm 4.0$ & $24.9 \pm 3.4$ & $24.5 \pm 4.3$ & 0.01 & 0.99 & 0.83 \\
\hline Pre-retrieval Serum Cr (mg/dL) & $1.8 \pm 1.4$ & $2.0 \pm 1.6$ & $2.1 \pm 0.7$ & 0.03 & 0.03 & 0.13 \\
\hline Serum $\mathrm{Cr}>2.0(\%)$ & $34(27.4)$ & $25(31.3)$ & $8(53.3)$ & 0.13 & & \\
\hline DM (\%) & $9(7.5)$ & $25(33.3)$ & $8(57.1)$ & $<0.01$ & $<0.01$ & 0.26 \\
\hline HTN (\%) & $18(15.0)$ & $40(53.3)$ & $6(42.9)$ & $<0.01$ & 0.04 & 0.99 \\
\hline CRRT (\%) & $16(14.9)$ & $12(17.1)$ & $2(13.3)$ & 0.95 & & \\
\hline Cause of death & & & & 0.03 & 0.04 & 0.33 \\
\hline CVA & $41(33.1)$ & $40(50.0)$ & $8(53.3)$ & & & \\
\hline Trauma & $26(21.0)$ & $15(18.8)$ & $6(40.0)$ & & & \\
\hline $\begin{array}{l}\text { Hypoxic } \\
\text { brain damage }\end{array}$ & $50(40.3)$ & $22(27.5)$ & $1(6.7)$ & & & \\
\hline unknown & $7(5.7)$ & $3(3.8)$ & $0(0)$ & & & \\
\hline KDPI & $52.4 \pm 22.5$ & $91.6 \pm 8.4$ & $99.6 \pm 0.6$ & $<0.01$ & $<0.01$ & $<0.01$ \\
\hline KDRI & $1.1 \pm 0.2$ & $1.8 \pm 0.4$ & $2.5 \pm 0.4$ & $<0.01$ & $<0.01$ & $<0.01$ \\
\hline
\end{tabular}

DKT group were $74.5 \mathrm{yrs} ., 24.5 \mathrm{~kg} / \mathrm{m}^{2}$, and $2.1 \mathrm{mg} / \mathrm{dL}$, respectively which represented the highest values among groups $(p<0.01,0.01$, and 0.03$)$. More donors of the ECD and DKT groups had diabetes or hypertension $(p<0.01$, both) and died from cerebrovascular disease $(p=0.03)$. The kidney donor profile index (KDPI) and kidney donor risk index (KDRI) were highest in the DKT group $(p<0.01$, both). Recipient characteristics are summarized in Table 2. The mean recipient age was 63.7 yrs. in the DKT group, which was the highest among the groups $(p<0.01)$. More diabetic recipients underwent DKT $(p<0.01)$. Patients with a previous history of $\mathrm{KT}$, panel reactive antigen (PRA) over $50 \%$, or DSAs were more prevalent in the SCD group $(p=$ $0.02,0.03$, and 0.02 , respectively). Mean cold ischemic times were not different among groups. Mean dialysis duration before KT in the DKT group ( 4.8 years) was shorter than in the ECD group (6.1 years), although the difference was not statistically significant $(p=0.17)$.

\section{Clinical outcomes}

Clinical outcomes are summarized in Table 3. Patient survival rates and death-censored graft survival rates were not different among groups (Fig. 1). Three years

Table 2 Recipient characteristics

\begin{tabular}{|c|c|c|c|c|c|c|}
\hline & $\operatorname{SCD}(n=124)$ & $\mathrm{ECD}(n=80)$ & DKT $(n=15)$ & $p$ value & SCD vs DKT & ECD vs DKT \\
\hline Age (year) & $49.3 \pm 10.1$ & $55.2 \pm 10.1$ & $63.7 \pm 6.7$ & $<0.01$ & $<0.01$ & $<0.01$ \\
\hline $\operatorname{Sex}(M: F)$ & $80: 44$ & $57: 23$ & $13: 2$ & 0.19 & & \\
\hline Dilaysis duration (yrs) & $6.6 \pm 4.5$ & $6.1 \pm 3.3$ & $4.8 \pm 2.5$ & 0.17 & & \\
\hline History of KT (\%) & $24(19.4)$ & $6(7.5)$ & $0(0)$ & 0.02 & 0.15 & 0.99 \\
\hline BMI & $23.0 \pm 3.5$ & $23.6 \pm 2.8$ & $22.6 \pm 2.9$ & 0.06 & & \\
\hline DM (\%) & $22(17.7)$ & $28(35.0)$ & $12(80.0)$ & $<0.01$ & $<0.01$ & $<0.01$ \\
\hline HTN (\%) & $97(78.2)$ & $70(87.5)$ & $11(73.3)$ & 0.16 & & \\
\hline HLA mm ${ }^{a}$ & $3(0-6)$ & $4(0-6)$ & $4(2-6)$ & $<0.01$ & 0.03 & 0.59 \\
\hline PRA $>50 \%$ & $25(20.8)$ & $6(7.5)$ & $1(6.7)$ & 0.03 & 0.55 & 0.99 \\
\hline DSA (+) & $21(17.2)$ & $5(6.25)$ & $0(0)$ & 0.02 & 0.25 & 0.99 \\
\hline CIT (min) & $287.9 \pm 83.6$ & $281.7 \pm 89.5$ & $290.3 \pm 107.0$ & 0.90 & & \\
\hline Induction agent (rATG:basiliximab:rituximab) & $79: 27: 18$ & $71: 6: 3$ & 15:0:0 & $<0.01$ & 0.03 & 0.99 \\
\hline
\end{tabular}

KT kidney transplantation, BMI body mass index, DM diabetes mellitus, HTN hypertension, HLA human leukocyte antigen, PRA panel reactive antibody, DSA donor specific antibody, CIT cold ischemic time

a Median (range), 
Table 3 Clinical outcomes

\begin{tabular}{|c|c|c|c|c|c|c|}
\hline & $\operatorname{SCD}(n=124)$ & $\mathrm{ECD}(n=80)$ & DKT $(n=15)$ & $p$ value & SCD vs DKT & ECD vs DKT \\
\hline Patient death (\%) & $4(3.2)$ & $3(3.8)$ & $0(0)$ & 0.96 & & \\
\hline Graft failure (\%) & $4(3.2)$ & $2(2.5)$ & $1(6.67)$ & 0.63 & & \\
\hline DGF (\%) & $33(26.6)$ & 27 (33.8) & $3(20.0)$ & 0.41 & & \\
\hline Nadir sCr & $1.2 \pm 0.7$ & $1.3 \pm 0.4$ & $1.0 \pm 0.3$ & $<0.01$ & 0.10 & $<0.01$ \\
\hline Time to nadir sCr & $24.6 \pm 30.0$ & $33.8 \pm 34.9$ & $22.6 \pm 21.3$ & 0.02 & 0.47 & 0.04 \\
\hline \multicolumn{7}{|l|}{ Post-transplant eGFR } \\
\hline $1 \mathrm{yr}$ & $60.2 \pm 15.0$ & $48.3 \pm 13.9$ & $57.0 \pm 21.5$ & $<0.01$ & 0.58 & 0.43 \\
\hline $2 \mathrm{yr}$ & $64.7 \pm 18.3$ & $53.1 \pm 14.8$ & $46.1 \pm 17.4$ & $<0.01$ & 0.03 & 0.23 \\
\hline $3 \mathrm{yr}$ & $65.8 \pm 18.0$ & $55.7 \pm 17.1$ & $50.1 \pm 19.1$ & 0.01 & 0.15 & 0.99 \\
\hline Rejection episode & $40(32.3)$ & $31(38.8)$ & $6(40.0)$ & 0.59 & & \\
\hline Complications $^{\mathrm{a}}(\%)$ & $6(4.8)$ & 19 (23.8) & $2(13.3)$ & $<0.01$ & 0.24 & 0.99 \\
\hline F/U duration (mo) & $33.5 \pm 15.0$ & $28.5 \pm 13.9$ & $25 \pm 12.1$ & $<0.01$ & 0.03 & 0.39 \\
\hline
\end{tabular}

DGF delayed graft function, $s C r$ serum creatinine level, eGFR estimated glomerulus filtration rate, $F / U$ follow up

${ }^{a}$ Complications include ureter leakage, ureter stricture, lymphocele, bleeding, and renal artery stenosis

after $\mathrm{KT}$, patient survival was $96.2 \%$ in the SCD group, $96.2 \%$ in the ECD group, and $100 \%$ in the DKT group. Death-censored graft survival 3 years after KT was $96.6 \%$ in the SCD group, $95.9 \%$ in the ECD group, and 100\% in the DKT group. There was one graft failure, which occurred in the DKT group. The graft dysfunction was attributed to diabetic nephropathy detected three years after KT, and HD was initiated six months later. Specifically, the recipient had diabetes, but the donor did not. The rate of DGF after DKT (20\%) was comparable to that of single SCD KT (26.6\%) and was lower than that of single ECD KT (33.8\%); however, the differences were not statistically significant $(p=0.41)$. In terms of nadir $\mathrm{sCr}$ level and time to nadir sCr, DKT was comparable to single SCD KT and superior to single ECD KT $(p<0.01$ and 0.02). Post-transplant eGFR one year after KT was lowest in the ECD group $(p<0.01)$. At two and three years after $\mathrm{KT}$, eGFRs were lowest in the DKT group $(p<0.01$ and 0.01); however, the eGFR and $\mathrm{sCr}$ trends were not significantly different among groups (Fig. 2,
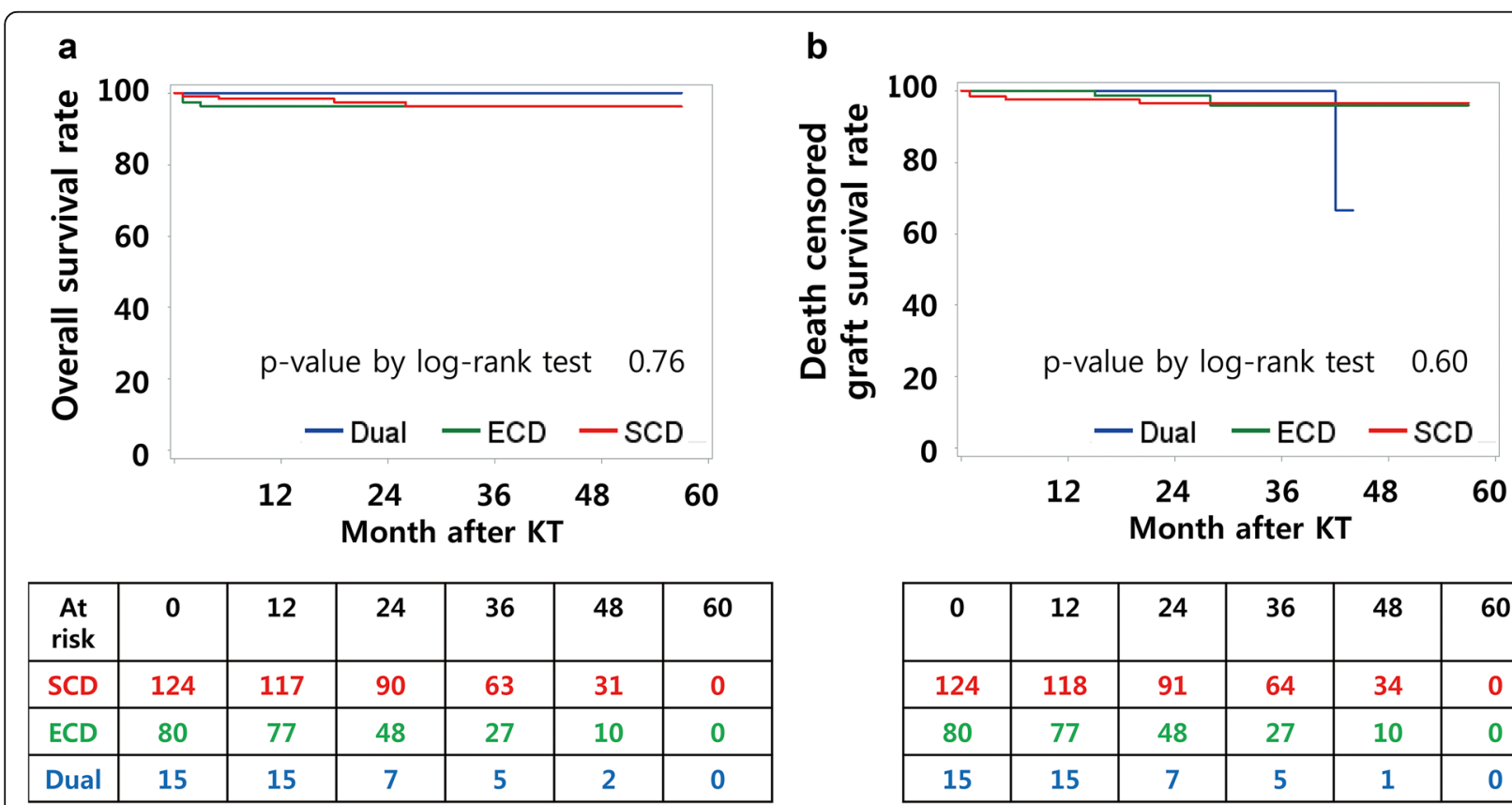

Fig. 1 Overall survival and death censored graft survival curves. (a) 3 years after KT, patient survival was 96.2\% in SCD group, 96.2\% in ECD group, and 100\% in DKT group. (b) Death censored graft survival at 3 years after KT was $96.6 \%$ in SCD group, 95.9\% in ECD group, and 100\% in DKT group

\begin{tabular}{|c|c|c|c|c|c|}
\hline 0 & 12 & 24 & 36 & 48 & 60 \\
\hline 124 & 118 & 91 & 64 & 34 & 0 \\
\hline 80 & 77 & 48 & 27 & 10 & 0 \\
\hline 15 & 15 & 7 & 5 & 1 & 0 \\
\hline
\end{tabular}




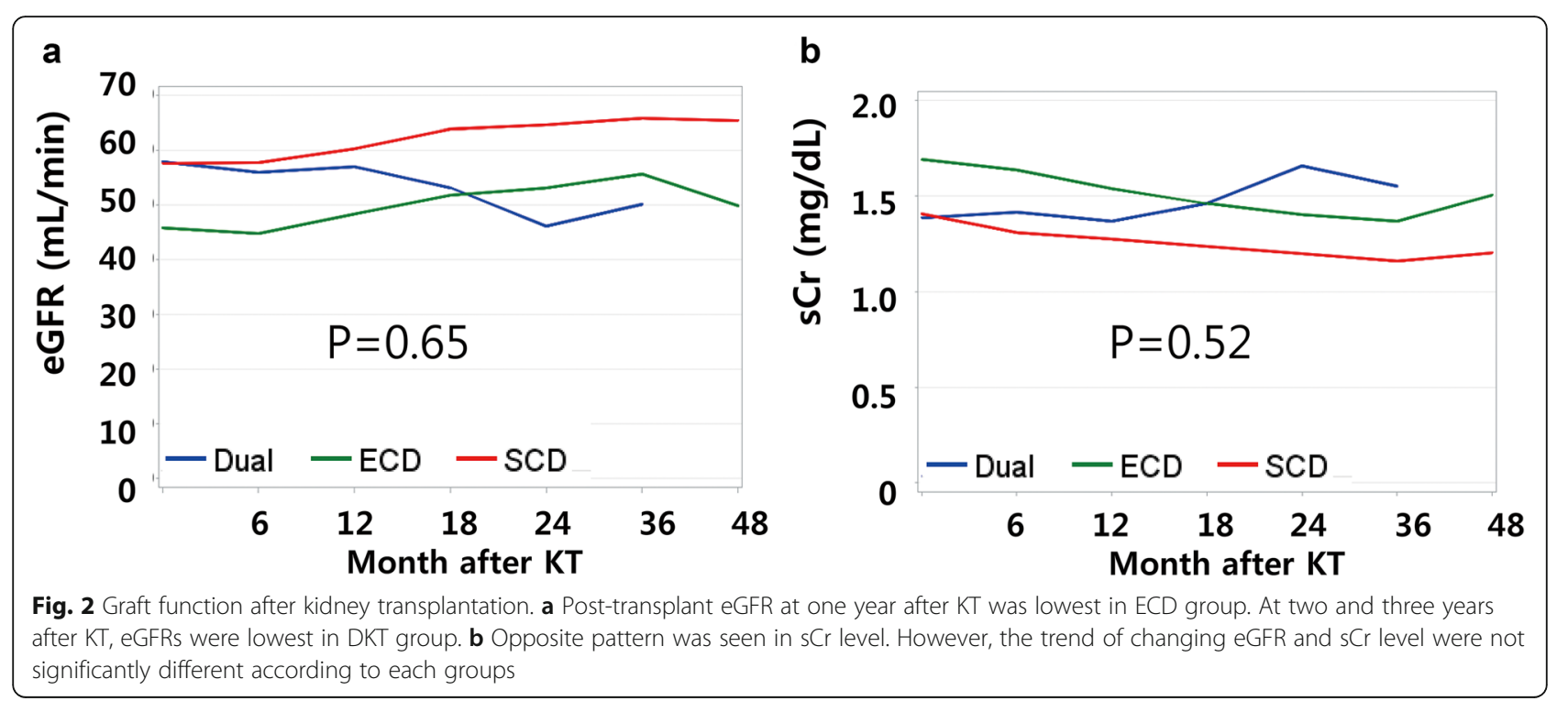

$p=0.65$ and 0.52 , respectively). The post-operative complication rate after DKT (13.3\%) was not higher than that after single ECD KT $(23.8 \%, p=0.99)$. There was one lymphocele and one ureteral leakage in the DKT group. The lymphocele was controlled after percutaneous drainage, while the ureteral leakage was controlled after double J stent and Foley catheter insertion, which were removed two and four weeks later, respectively.

\section{Discussion}

Outcomes of DKT in our study were not different from those of single KTs in terms of graft survival rate and graft function after $\mathrm{KT}$ despite a higher age, higher $\mathrm{sCr}$ level, greater burden of diabetes, and higher KDPI and KDRI scores in DKT donors $(p<0.01$ in all). Disadvantages from the donor factors were overcome by doubling the number of transplanted nephron in DKT. Even though the difference was not statistically significant $(p=0.41)$, the rate of DGF after DKT $(20 \%)$ was lower than that of single ECD KT $(33.8 \%)$. It can be explained by that DKT can supply sufficient number of nephron and, even if some fraction of nephrons were injured, enough number of nephrons is preserved to facilitate primary function.

Recently, many studies have reported that graft survival and graft function are not significantly difference between single KT and DKT [5-10, 13-20]. However, the donor selection criteria for DKT among these studies varies. Most studies have used histology based selection criteria such as the 12-point Kalpinski system or the Remuzzi scoring system [5-7, 13-15, 17-19]. In a clinical setting not supported by sufficient pathologists and without a centralized donor management system, scoring of donor kidney biopsy specimens is nearly impossible. Therefore, in our study, we used objective clinical values such as donor age, eGFR, and $\mathrm{sCr}$ level as the donor selection criteria for DKT.

KONOS data indicated that the kidney discard rate over the last decade in Koreas was higher among donors aged more than $70(18.1 \%)$ compared to donors younger than 70 (7.4\%). Additionally the discard rate in donors between 60 and 70 years was 9.6\%. In a clinical setting with insufficient support by pathologists specialized in kidney allograft histology, donor age is the most important factor in the decision of a clinician to discard a kidney graft. Therefore, to reduce the graft discard rate it is necessary to use kidneys from older donors. Numerous studies have reported successful outcomes after DKT from donors older than $70[7,14,16-20]$. Thus, we selected 70 as the donor age threshold in our study. The mean donor age in our study was 74.5 yrs. Two donors were younger than 70 (ages 66 and 69), and both had kidneys denied from all other centers.

Even though our results were comparable to the results of single ECD KT, there should be criteria for clinicians to decline a graft kidney. We discarded kidneys when they were grossly discolored or atrophied, or when the donor $\mathrm{sCr}$ level had slowly and consistently deteriorated for a period longer than 5 days. Nevertheless, we agree that a histological scoring system is more objective if pathological assessment of the donor kidney is available. Further efforts to establish pathology support systems for kidney graft evaluation are thus necessary.

Our center utilized an "old for old" strategy. As a result, the mean age of recipients in the DKT group (63.7 yrs) was significantly higher than in the ECD group (55.2 yrs., $p<0.01$ ). In our study, the eGFR level one year after KT was higher in the DKT group than in the ECD group. However, we found that this situation reversed at two and three years after KT. Although the trend of 
eGFR change was not significantly different in these groups $(p=0.65)$, it seems reasonable and prudent to transplant kidneys with the potential to last longer into recipients who are expected to live longer. Moreover, the DKT group showed a lower rate of DGF, a lower level of nadir $\mathrm{sCr}$, and a shorter duration to nadir $\mathrm{sCr}$, all of which can lower recipient's physical burden during the immediate post-operative period. These factors can be helpful, especially for older recipients with less physical reservoir. The duration of dialysis prior to KT was shorter in the DKT group ( 4.8 years) than in the ECD group (6.1 years), although the difference was not statistically significant $(p=0.17)$. Considering the age of older recipients and the importance of performing $\mathrm{KT}$ as soon as possible, DKT should be viewed as a reasonable solution. In addition, Rigotti et al. improved DKT outcomes for older recipients with a personalized immunosuppression strategy [17]. Although we did not utilize this approach, use of mTOR inhibitor to lower doses of calcineurin inhibitors represents a potential way to improve outcomes [17].

There were several limitations to the present study. The number of DKT cases was relatively small and the follow-up duration was too short to draw long-term results. Given these limitations, the ability to generalize our results may be limited. Despite these limitations, this study is valuable because of the successful utilization of kidneys from extremely marginal donors for DKT. To the best of our knowledge, this is the first report of DKT outcomes in an Asian population. Out results also provide evidence for strategies to improve the use of kidneys safely from marginal donors.

\section{Conclusion}

DKT provides comparable graft survival and graft function to single ECD KT despite older donors with higher $\mathrm{sCr}$ levels, more diabetes, and higher KDPI and KDRI scores. DKT is a safe and feasible way to use kidney grafts from extremely marginal donors, thereby reducing the organ discard rate. Further efforts should focus on avoiding inappropriate use of unacceptable kidneys and improving DKT outcomes.

\footnotetext{
Abbreviations

DD: Deceased donor; DDKT: Deceased donor kidney transplantation; DGF: Delayed graft function; DKT: Dual kidney transplantation; DSA: Donorspecific ant-HLA antibody; ECD: Expanded criteria donor; eGFR: Estimated glomerular filtration; GEE: Generalized Estimating Equation; KDPI: Kidney donor profile index; KDRI: Kidney donor risk index; KONOS: Korean Network for Organ Sharing; KT: Kidney transplantation; PRA: Panel reactive antigen; SCD: Standard criteria donor; sCr: Serum creatinine; UNOS: United Network for Organ Sharing
}

Acknowledgements

Not applicable.
Ethics, consent and permissions

We did not use kidneys from prisoners. All kidneys were obtained from the Seoul national university hospital, Samsung Medical Center, and Chungbuk national university hospital. The institutional review board of Samsung Medical Center approved this retrospective study protocol (SMC 2018-03035) and waived the requirement for written informed consent.

\section{Authors' contributions}

KWL: Project development, data collection, data analysis, and manuscript writing. JBP: Project development and manuscript editing. SRC: Data collection. SHL: Data collection. YJC: Data collection. HY: Data analysis. KK: Data analysis. SJK: Project development and manuscript editing. All authors have read and approved the manuscript.

\section{Funding}

The authors have no relevant affiliations or financial involvement with any organization or entity with a financial interest in or financial conflict with the subject matter or materials discussed in the manuscript.

\section{Availability of data and materials}

The datasets used and/or analysed during the current study are available from the corresponding author on reasonable request.

\section{Consent for publication}

Not applicable.

\section{Competing interests}

The authors declare no potential conflicts of interest.

\section{Author details}

'Department of Surgery, Samsung Medical Center, Sungkyunkwan University School of Medicine, 81 Irwon-ro, Gangnam-gu, Seoul 06351, South Korea.

${ }^{2}$ Organ Transplantation Center, Samsung Medical Center, Seoul, South Korea.

${ }^{3}$ Statistics and Data Center, Samsung Medical Center, Seoul, South Korea.

Received: 19 April 2019 Accepted: 10 December 2019

Published online: 06 January 2020

\section{References}

1. Hwang JK, Park SC, Kwon KH, Choi BS, Kim JI, Yang CW, Kim YS, Moon IS. Long-term outcomes of kidney transplantation from expanded criteria deceased donors at a single center: comparison with standard criteria deceased donors. Transplant Proc. 2014;46(2):431-6.

2. Lionaki S, Kapsia H, Makropoulos I, Metsini A, Skalioti C, Gakiopoulou H, Zavos G, Boletis JN. Kidney transplantation outcomes from expanded criteria donors, standard criteria donors or living donors older than 60 years. Ren Fail. 2014;36(4):526-33.

3. Tanrisev M, Hoscoskun C, Asci G, Sozbilen M, Firat O, Ertilav M, Ozkahya M, Toz H. Long-term outcome of kidney transplantation from elderly living and expanded criteria deceased donors. Ren Fail. 2015;37(2):249-53.

4. Lee CM, Scandling JD, Shen GK, Salvatierra O, Dafoe DC, Alfrey EJ. The kidneys that nobody wanted: support for the utilization of expanded criteria donors. Transplantation. 1996:62(12):1832-41.

5. Remuzzi G, Grinyo J, Ruggenenti P, Beatini M, Cole EH, Milford EL, Brenner BM, DKTG DKG. Early experience with dual kidney transplantation in adults using expanded donor criteria. J Am Soc Nephrol. 1999;10(12):2591-8.

6. D'Arcy FT, O'Connor KM, Shields W, Zimmerman JA, Mohan P, Eng M, Little DM, Power R, Dorman A, Hickey DP. Dual kidney transplantation with organs from extended criteria cadaveric donors. J Urol. 2009:182(4):1477-81.

7. Ekser B, Furian L, Broggiato A, Silvestre C, Pierobon ES, Baldan N, Rigotti P. Technical aspects of unilateral dual kidney transplantation from expanded criteria donors: experience of 100 patients. Am J Transplant. 2010;10(9): 2000-7.

8. Mendel L, Albano L, Bentellis I, Yandza T, Bernardi C, Quintens H, Tibi B, Jourdan J, Durand M, Amiel J, Chevallier D. Safety of dual kidney transplantation compared to single kidney transplantation from expanded criteria donors: a single center cohort study of 39 recipients. Transpl Int. 2018:31(10):1110-24.

9. Stratta RJ, Farney AC, Orlando G, Farooq U, Al-Shraideh Y, Palanisamy A, Reeves-Daniel A, Doares W, Kaczmorski S, Gautreaux MD, Iskandar SS, Hairston G, Brim E, Mangus M, El-Hennawy H, Khan M, Rogers J. Dual kidney 
transplants from adult marginal donors successfully expand the limited deceased donor organ pool. Clin Transpl. 2016;30(4):380-92.

10. Moore PS, Farney AC, Sundberg AK, Rohr MS, Hartmann EL, Iskandar SS, Gautreaux MD, Rogers J, Doares W, Anderson TK, Adams PL, Stratta RJ. Dual kidney transplantation: a case-control comparison with single kidney transplantation from standard and expanded criteria donors. Transplantation. 2007;83(12):1551-6.

11. Metzger RA, Delmonico FL, Feng S, Port FK, Wynn JJ, Merion RM. Expanded criteria donors for kidney transplantation. Am J Transplant. 2003;3(Suppl 4):114-25.

12. Lee KW, Park JB, Cho CW, Lee N, Yoo H, Kim K, Park H, Kang ES, Huh W, Kim S. The impact of donor-specific anti-human leukocyte antigen (HLA) antibody rebound on the risk of antibody mediated rejection in sensitized kidney transplant recipients. Ann Transplant. 2017;22:166-76.

13. Remuzzi G, Cravedi P, Perna A, Dimitrov BD, Turturro M, Locatelli G, Rigotti P, Baldan N, Beatini M, Valente U, Scalamogna M, Ruggenenti P, Dual Kidney Transplant Group. Long-term outcome of renal transplantation from older donors. N Engl J Med. 2006;354(4):343-52.

14. De Serres SA, Caumartin Y, Noel R, Lachance JG, Cote I, Naud A, Fradet $Y$, Mfarrej BG, Agharazii M, Houde I. Dual-kidney transplants as an alternative for very marginal donors: long-term follow-up in 63 patients. Transplantation. 2010;90(10):1125-30.

15. Lucarelli G, Bettocchi C, Battaglia M, Impedovo SV, Vavallo A, Grandaliano G, Castellano G, Schena FP, Selvaggi FP, Ditonno P. Extended criteria donor kidney transplantation: comparative outcome analysis between single versus double kidney transplantation at 5 years. Transplant Proc. 2010;42(4):1104-7.

16. Gallinat A, Feldkamp T, Schaffer R, Radunz S, Treckmann JW, Minor T, Witzke O, Paul A, Sotiropoulos GC. Single-center experience with kidney transplantation using deceased donors older than 75 years. Transplantation. 2011;92(1):76-81.

17. Rigotti P, Capovilla G, Di Bella C, Silvestre C, Donato P, Baldan N, Furian L. A single-center experience with 200 dual kidney transplantations. Clin Transpl. 2014;28(12):1433-40.

18. Mallon DH, Riddiough GE, Summers DM, Butler AJ, Callaghan CJ, Bradbury LL, Bardsley V, Broecker V, Saeb-Parsy K, Torpey N, Bradley JA, Pettigrew GJ. Successful transplantation of kidneys from elderly circulatory death donors by using microscopic and macroscopic characteristics to guide single or dual implantation. Am J Transplant. 2015:15(11):2931-9.

19. De Paolis P, Colonnelli R, Favaro A, Salem F, Vignally P, Carriero C, lappelli M, Di Giulio S. Expanded criteria donor kidney transplantation: comparative outcome evaluation between single versus double kidney transplantation at 8 years: a single center experience. Transplant Proc. 2016;48(2):329-32.

20. Khalid U, Asderakis A, Rana T, Szabo L, Chavez R, Ilham MA, Ablorsu E. Dual kidney transplantation offers a valuable source for kidneys with good functional outcome. Transplant Proc. 2016:48(6):1981-5.

\section{Publisher's Note}

Springer Nature remains neutral with regard to jurisdictional claims in published maps and institutional affiliations.

Ready to submit your research? Choose BMC and benefit from:

- fast, convenient online submission

- thorough peer review by experienced researchers in your field

- rapid publication on acceptance

- support for research data, including large and complex data types

- gold Open Access which fosters wider collaboration and increased citations

- maximum visibility for your research: over $100 \mathrm{M}$ website views per year

At BMC, research is always in progress.

Learn more biomedcentral.com/submissions 